\title{
Effect of once-daily FDC treatment era on initiation of cART
}

\author{
This article was published in the following Dove Press journal: \\ HIVIAIDS - Research and Palliative Care \\ II February 2010 \\ Number of times this article has been viewed
}

\author{
David M Mosen' \\ Michael Horberg² \\ Douglas Roblin ${ }^{3}$ \\ Christina M Gullion' \\ Richard Meenan' \\ Wendy Leyden ${ }^{2}$ \\ Weiming $\mathrm{Hu}^{\prime}$ \\ 'Center for Health Research, \\ Kaiser Permanente Northwest, \\ Portland, OR, USA; '2Division of \\ Research, Kaiser Permanente \\ Northern California, Oakland, \\ CA, USA; ${ }^{3}$ Center for Health \\ Research, Kaiser Permanente \\ Georgia, Atlanta, GA, USA
}

Correspondence: David Mosen 3800 N. Interstate Avenue, Portland, OR 97227-I I I0, USA

Tel +I 5033356637

$\mathrm{Fax}+\mathrm{I} 5033352424$

Email david.m.mosen@kpchr.org
Objectives: Combination antiretroviral therapy (cART) is associated with increased survival among HIV-infected persons. Yet, no research to date has examined whether introduction of once-daily fixed-dosed combinations (FDC) affects the likelihood of cART initiation. We aimed to determine whether implementation of once-daily FDC regimens was associated with changes to cART initiation. We also identified clinical, treatment regimen, and provider characteristics possibly associated with cART initiation.

Study design: Retrospective observational analysis.

Methods: We queried electronic medical records between July 1999-June 2006 to identify incident cases of detectable HIV infection in antiretroviral-naïve adults. Cox regression with time-dependent covariates was used to examine the effects of once-daily FDC era, clinical, provider, and treatment regimen characteristics on cART initiation.

Results: Once-daily FDC availability did not change the likelihood of cART initiation, but other characteristics were associated with an increased likelihood: AIDS diagnosis, above-median daily pill consumption, and 16+ yrs of physician HIV experience. Decreased likelihood of cART initiation was associated with CD4 201-350 cells/ $\mu \mathrm{L}$, HIV RNA $<100,000$ copies/mL, and with CD4 $>350$ cells $/ \mu \mathrm{L}$ (any HIV RNA level), compared to CD4 $\leq 200$ cells $/ \mu \mathrm{L}$.

Conclusion: Availability of once-daily FDC-based regimens did not affect likelihood of cART initiation. Patient clinical characteristics appear to be more important predictors of cART initiation.

Keywords: ARV treatment, once-daily FDC therapies, ARV-naïve

\section{Introduction}

In the 1990s, when combination antiretroviral therapy (cART) was developed, treatment regimens involved multiple pills, dosages, and potentially complex drug-drug interactions. ${ }^{1,2}$ However, these regimens were also successful, so simplifying them was a priority. ${ }^{3,4}$ Since 2005 , physicians have been able to prescribe combination antiretroviral (ARV) regimens as simple as one pill daily, and patient-level data indicate preference for these easier-to-follow regimens. ${ }^{5,6}$ What is not clearly understood, however, is whether regimen simplification is independently associated with initiation of cART, irrespective of clinical factors such as CD4 count and human immunodeficiency virus (HIV) RNA levels identified in HIV treatment guidelines. ${ }^{7,8}$ Such research is important to better understand whether adoption of new treatment regimens may unduly affect clinician prescribing practices, independent of predictors identified in HIV treatment guidelines.

Early studies by Kitahata ${ }^{9}$ indicated that more experienced HIV providers were more likely to initiate cART earlier, and to follow established HIV care guidelines.

submit your manuscript | www.dovepress.com 
By late 1990s, HIV care guidelines advocated a "hit early, hit hard" philosophy. ${ }^{1,2}$ However, as research came to light that ARV therapy could eventually produce accumulated adverse effects, HIV care providers became more reluctant to initiate ARV therapy when CD4 T-cell counts were greater than 350 cells $/ \mu \mathrm{L}$, with many preferring to wait until the patient's CD4 count dropped to $<200$ cells $/ \mu \mathrm{L} .{ }^{10} \mathrm{HIV}$ care guidelines from the early 2000s reflected this reluctance. ${ }^{3,4}$ Now that once-daily fixed-dose combination (FDC) therapies are available, as are therapies with fewer adverse effects, many researchers are suggesting that a more aggressive approach to ARV-naïve patients is in order, particularly in light of studies showing better long-term outcomes with earlier initiation of therapy. ${ }^{11-14}$

Clearly, research is lacking on how recent developments in ARV therapy, especially FDC therapies and fewer dosages daily, have affected HIV care providers' prescribing habits in regards to cART initiation. Therefore, our study on cART initiation among ARV-naïve (never exposed to ARV medications) HIV-infected patients had two primary objectives: 1) to determine whether initiation of cART changed before and after the introduction of once-daily FDC therapies into clinical practice; and, 2) to identify patient clinical characteristics, provider characteristics, and/or treatment regimen characteristics associated with cART initiation after adjusting for implementation of once-daily FDC therapies into clinical practice. Kaiser Permanente (KP), a multi-state integrated healthcare system, is ideally suited to study such potential prescribing changes over time.

\section{Methods}

\section{Study population}

We queried the electronic medical record (EMR) databases of three KP regions: Northern California, Northwest (ie, metropolitan Portland, Oregon), and Georgia (ie, metropolitan Atlanta) to identify adult HIV-infected ARV-naïve patients (males and females aged $\geq 18$ years old). KP is an integrated health care system serving over eight million individuals in the United States. Together, these three regions represent $45 \%-50 \%$ of KP's HIV population.

The study observation period was from July 1st, 1999 through June 30th, 2006. An ARV-naïve patient was defined by the following inclusion and exclusion criteria:

1. Laboratory results showing detectable HIV RNA (defined as $\geq 75$ copies/mL of HIV RNA) on a report generated between July 1st, 1999 through June 30th, 2006 (the index date), but not before July 1st, 1999 (ie, new treatment candidates).
2. One hundred eighty three or more days of KP health plan coverage (including KP pharmacy benefits) prior to the index date (the start date, earliest was January 1st, 1999; latest was December 31st, 2005).

3. One hundred eighty three or more days of KP health plan coverage (including KP pharmacy benefits) after the index date.

4. ARV-naïve: no history of ARV treatment in the 183 days prior to the index date.

All patients were followed for a minimum of 183 days after the index date until the earliest of the following: 1) initiation of first cART regimen (event date, our primary outcome); 2) death; 3 ) end of observation period (December 31 st, 2006); or 4) start of first health plan disenrollment lasting four or more months. The date associated with any such event was the exit date. If cART recipients received one or more cART regimens during the follow-up period, only the first regimen was counted as an event. Cohort members who never received a cART regimen during the follow-up period (through December 31st, 2006) had a censored outcome at their exit date.

\section{Data sources and measures} Data sources

Appropriate pharmacy, laboratory, utilization, membership eligibility, and provider employment databases in each respective KP region were queried to obtain outcome and predictor data elements between January 1st, 1999 and December 31st, 2006. We searched records for diagnostic or treatment evidence of four co-morbidities dating back to 1995: coronary artery disease (CAD), diabetes mellitus (DM), active hepatitis B (HBV), and hepatitis C (HCV).

\section{Outcome measure}

The primary outcome measure was time to event of first pharmacy dispensing of cART after the index date. cART was defined as $\geq 3$ ARVs that met one of the acceptable combinations, based on 2006 DHHS treatment guidelines. ${ }^{15}$

\section{Primary independent variable}

The once-daily FDC time era was the primary independent variable in this study, and was treated as a time-dependent indicator (pre- vs post-FDC eras). The pre-FDC era included dates between July 1st, 1999 and December 31st, 2004, while the post-FDC era was defined as January 1st, 2005 through June 30th, 2006. We selected January 1st, 2005 as the start date of the post-FDC era, because once-daily FDC treatment regimens were implemented within KP from this 
date (January 1st, 2005) forward. Patients who contributed information in both eras were represented by at least two records in the time-dependent dataset. Although twice-daily FDC treatment regimens were used in clinical practice beginning in 1998 within KP, we focused on the once-daily FDC treatment period (starting in January 1st, 2005) because of the potentially larger impact on cART initiation.

\section{Secondary independent variables}

Secondary independent variables included clinical, provider, and treatment-regimen characteristics. Clinical variables collected were: 1) CD4 T-cell count; 2) HIV RNA level; 3 ) an indicator (yes/no) of whether patient met criteria for AIDS diagnosis (1993 CDC Classification); 4) an indicator of CAD or DM diagnosis; and 5) an indicator of $\mathrm{HCV}$ and/ or HBV diagnosis. Values for each measure were assessed as close as possible to the index date and then updated throughout the study period as clinical values changed. A CD4 T-cell count/HIV RNA composite measure was defined using 6 levels: 1) CD4 $\leq 200$ cells/ $\mu \mathrm{L}$ and HIV RNA $\geq 100,000$ copies/mL; 2 ) CD $4 \leq 200$ cells/ $\mu \mathrm{L}$ and HIV RNA $<100,000$ copies/mL; 3) CD4 201-350 cells/ $\mu \mathrm{L}$ and HIV RNA $\geq 100,000$ copies/mL; 4) CD4 201-350 cells/ $\mu \mathrm{L}$ and HIV RNA $<100,000$ copies/mL; 5) CD4 $>350$ cells $/ \mu \mathrm{L}$ and HIV RNA $<100,000$ copies/mL; and 6) CD4 > 350 cells/ $\mathrm{uL}$ and HIV RNA $\geq 100,000$ copies/mL. This six-level classification measure was based on previous cutpoints established in the HIV literature that examined CD4 and HIV RNA levels associated with mortality.,16

Provider characteristics were: 1) years caring for HIV patients within the KP system; 2) HIV panel size per clinician; and 3) medical specialty (HIV specialist with infectious disease certification, HIV specialist, noninfectious disease certification, or general medicine). This information was collected as close as possible to index date and updated annually throughout the follow-up period, if a provider characteristic had changed, or if a patient changed provider in the previous year. We collapsed years spent caring for HIV patients into a four-level ordinal variable $(0-5$ years, 6-11 years, $12-16$ years, $>16$ years), and did the same with HIV panel size ( $\leq 50,51-100,101-200,201+)$.

Treatment regimen characteristics included: 1) patient average daily total pill count, and 2) region-wide median daily ARV pill count. These counts represent patient burden of overall pill-taking between the start and exit date (not time-dependent), as well as the burden of HIV treatment for all patients, at the same period in the same region as a given patient, respectively.
Average daily pill count for a patient was calculated as the sum of all prescriptions (pills per day multiplied by the number of days' supply) divided by the number of days between the start and exit dates. For example, the average daily pill count for a patient with 365 days of follow-up time that had 18 total dispensings, with each dispensing taken twice per day for a total of 30 days (days' supply) would be as follows: $18 \times[(2 \times 30)] / 365=2.96$ pills per day. Only oral pill-based medications were included. For analysis, the measure is dichotomized at the median overall patients in the analysis sample.

Aggregate, region-based ARV pill count is defined as the median daily count of cART pills dispensed among all $\mathrm{HIV}+$ patients in that patient's region (ie, not restricted to patients included in this study), upon the first dispensing that met cART criteria. Rather than a patient-based average of all medications between the start and exit date, the region-based pill-count measure assessed the median daily pill count of ARV medications upon the first cART dispensing among all patients within the study region that qualified for cART. To more precisely measure changes in regional cART dispensing practices over time, the measure was updated every six months (January 1st-June 30th, July 1 st-December $31 \mathrm{st}$ ) between a patient's start-to-exit dates, so this variable was time-dependent. For example, if a patient had an index date of March 15th, 2001, the regionbased ARV pill count for that patient would be the value for the patient's region for January 1st-June 30th, 2001. Because this measure was region-, rather than patient-based, patients with index dates between January 1st-June 30th or July 1st-Dec 31 st would have identical region-based ARV pill count values. For analysis, the region-based ARV pill-count measure was also dichotomized at the median (calculated over all regions).

\section{Control variables}

Five background variables served as control variables: 1) age at index date; 2) sex; 3 ) race/ethnicity (white, African American, Hispanic, Asian-American, other/unknown); 4) an indicator of injection drug use (IDU) status (yes vs no/ unknown); and 5) KP region location (Northern California, Northwest, Georgia).

\section{Analysis}

Cox proportional-hazards regression with time-dependent covariates was used to estimate the relative hazard of cART initiation as a function of risk profile, where risk profile was defined as a combination of provider, clinical, and 
treatment regimen characteristics. To check whether the proportional hazards assumption was violated, we estimated the Kaplan-Meier survival density function (SDF) for each independent variable, as well as the plot of log time vs log negative log of the SDF. If curves crossed or were overlaid, we made adjustments to the category definition for that variable. To reduce proportional hazards violation, HIV panel size was dichotomized $(0-100,101+)$. We found that CAD/DM could be eliminated because the SDF for positive and negative values was the same. Finally, the number of categories for the CD4 T-cell count/HIV RNA composite could be reduced to five by combining the two CD $4 \leq 200$ cells $/ \mu \mathrm{L}$ categories, which had the same SDF.

Last, we used Cochran-Mantel-Haenszel tests of general association to check for collinearity between pairs of independent variables. Because we found that provider specialty was strongly associated with panel size $(P<0.0001)$, as well as violated the proportional hazards assumption, we dropped it from the variable list.

We obtained approval from all regional KP Institutional Review Boards. The Institutional Review Boards waived the requirement for informed consent prior to the start of the study for all patients.

\section{Results}

\section{Baseline study characteristics}

A total of 2,127 patients met initial inclusion criteria. Of these, 1,662 (78.1\%) had an index date in the pre-FDC era, while 465 $(21.9 \%)$ had an index date in the post-FDC era (Tables 1,2$)$.

\section{Demographic characteristics}

Nearly $90 \%$ of patients were male, with a mean age close to 40 years (Table 1). About half of these patients were nonCaucasian. Mirroring the national KP population, more than two-thirds of patients were identified from the (Northern) California region. Fewer than $6 \%$ had records indicating history of injection drug use as an HIV risk behavior. The distribution of race/ethnicity appears to be significantly different across the two FDC-time eras, primarily due to an increase in other/ unknown category. No other demographic differences were noted between the pre- and post-once-daily FDC eras.

\section{Clinical characteristics}

As of index date, nearly $40 \%$ of the population had CD 4 $\leq 200$ cells $/ \mu \mathrm{L}$, while about one-third had CD4 $>350$ cells $/ \mu \mathrm{L}$ and HIV RNA $\geq 100,000$ copies/mL (Table 2). About 34\% met AIDS criteria. Fewer than $5 \%$ had documentation of active

Table I Descriptive analysis: patient demographic characteristics

\begin{tabular}{|c|c|c|c|c|}
\hline Demographics & $\begin{array}{l}\text { Overall } \\
N=2,127\end{array}$ & $\begin{array}{l}\text { Pre-FDC era' } \\
N=1,662\end{array}$ & $\begin{array}{l}\text { Post-FDC era } \\
N=465\end{array}$ & $\mathbf{P}^{*}<$ \\
\hline Age (mean $\pm S D)$ & $41.1 \pm 9.8$ & $41.0 \pm 9.7$ & $41.2 \pm 10.4$ & NS** \\
\hline Male: n (\%) & $\mathrm{I}, 876(88.2)$ & $\mathrm{I}, 45 \mathrm{I}(87.3)$ & $425(91.4)$ & NS** \\
\hline Race/Ethnicity: n (\%) ${ }^{3}$ & & & & 0.0001 \\
\hline White & $996(46.8)$ & $780(46.9)$ & $216(46.5)$ & \\
\hline African American & $518(24.4)$ & $423(25.5)$ & $95(20.4)$ & \\
\hline Hispanic & $27 \mid(12.7)$ & $226(13.6)$ & $45(9.7)$ & \\
\hline Asian American & $93(4.4)$ & $68(4.1)$ & $25(5.4)$ & \\
\hline Other/Unknown & $249(11.7)$ & $165(9.9)$ & $84(18.1)$ & \\
\hline IDU risk category: $\mathrm{n}(\%)^{3}$ & & & & NS** \\
\hline Yes & $|2|(5.7)$ & $97(5.8)$ & $24(5.2)$ & \\
\hline No & $\mathrm{I}, 046(49.2)$ & $827(49.8)$ & $219(47.1)$ & \\
\hline Information not available & $960(45.1)$ & $738(44.4)$ & $222(47.7)$ & \\
\hline KP region location: $\mathrm{n}(\%)^{3}$ & & & & NS** \\
\hline Georgia & $409(19.2)$ & $313(18.8)$ & $96(20.7)$ & \\
\hline Northern California & $\mathrm{I}, 546(72.7)$ & $\mathrm{I}, 2 \mathrm{I} 2(72.9)$ & $334(71.8)$ & \\
\hline Northwest & $172(8.1)$ & 137 (8.2) & $35(7.5)$ & \\
\hline
\end{tabular}

*Test that baseline characteristics differed between pre-FDC and post-FDC eras, 2-tailed alpha $=0.05$.

**“NS" (not significant), defined as $P>0.05$.

'Includes index dates $\geq 7 / 1 / 1999$ and $\leq 12 / 31 / 2004$.

${ }^{2}$ Includes index dates $\geq 1 / 1 / 2005$ and $\leq 6 / 30 / 2006$.

${ }^{3}$ Column percentages may not add up to $100 \%$ due to rounding.

Abbreviations: FDC, fixed dose combination; IDU, injection drug use; KP, Kaiser Permante. 
Table 2 Descriptive analysis: baseline clinical, provider, and treatment regimen characteristics

\begin{tabular}{|c|c|c|c|c|}
\hline Characteristic & $\begin{array}{l}\text { Overall } \\
\mathbf{N}=2,127\end{array}$ & $\begin{array}{l}\text { Pre-FDC era' } \\
\mathbf{N}=1,662\end{array}$ & $\begin{array}{l}\text { Post-FDC era }{ }^{2} \\
\mathbf{N}=465\end{array}$ & $\mathbf{P}^{*}<$ \\
\hline \multicolumn{5}{|l|}{ Clinical } \\
\hline \multicolumn{5}{|l|}{$\mathrm{n},(\%)^{3,4}$} \\
\hline$C D 4 \leq 200$ & $786(37.0)$ & $653(39.3)$ & I $33(28.6)$ & \\
\hline CD4 20I-350 and HIV RNA $\geq 100 \mathrm{~K}$ & $396(18.6)$ & $313(18.8)$ & $82(17.6)$ & \\
\hline CD4 20I-350 and HIV RNA $<100 \mathrm{~K}$ & III (5.2) & $83(5.0)$ & $28(6.0)$ & \\
\hline $\mathrm{CD} 4>350$ and $\mathrm{HIV} \mathrm{RNA} \geq 100 \mathrm{~K}$ & $737(34.7)$ & $537(32.3)$ & $200(43.0)$ & \\
\hline $\mathrm{CD} 4>350$ and $\mathrm{HIV}$ RNA $<100 \mathrm{~K}$ & $98(4.6)$ & $76(4.6)$ & $22(4.7)$ & \\
\hline AIDS criteria; $\mathrm{n}(\%)^{5}$ & $731(34.4)$ & $591(35.6)$ & $140(30.1)$ & 0.05 \\
\hline Active $\mathrm{HBV}$ and/or HCV; $\mathrm{n}(\%)^{5}$ & $91(4.3)$ & $75(4.5)$ & $16(3.4)$ & NS** \\
\hline \multicolumn{5}{|l|}{ Provider } \\
\hline \multicolumn{2}{|l|}{ Years caring for HIV patients: $\mathrm{n}(\%)^{3,6}$} & & & 0.0001 \\
\hline $0-5$ & $392(18.4)$ & $254(15.3)$ & I $58(29.7)$ & \\
\hline $6-11$ & $527(24.8)$ & $451(27.1)$ & $76(16.3)$ & \\
\hline $12-16$ & $793(37.3)$ & $704(42.4)$ & $89(19.1)$ & \\
\hline$>16$ & $383(18.0)$ & $227(13.7)$ & $156(33.6)$ & \\
\hline Information not available & $32(1.5)$ & $26(1.6)$ & $6(1.3)$ & \\
\hline \multicolumn{2}{|l|}{ HIV panel size: $\mathrm{n}(\%)^{3,6}$} & & & NS** \\
\hline$\leq 100$ & $882(4 \mid .5)$ & $685(4 \mid .2)$ & $197(42.4)$ & \\
\hline $101+$ & $1213(57.0)$ & $95 I(57.2)$ & $262(56.3)$ & \\
\hline Information not available & $32(1.5)$ & $26(1.6)$ & $6(1.3)$ & \\
\hline \multicolumn{5}{|l|}{ Treatment regimen } \\
\hline Total pill count per day (median, IQR) ${ }^{7}$ & $1.7,0.6-3.2$ & $1.8,0.7-3.4$ & $1.3,0.4-2.8$ & 0.01 \\
\hline Region-based ARV pill count per day (median, IQR) ${ }^{8}$ & $13.0,6.0-22.0$ & $18.0,11.0-22.0$ & $6.0,6.0-7.0$ & $<0.0001$ \\
\hline \multicolumn{5}{|c|}{$\begin{array}{l}\text { *Test that baseline characteristics differed between pre-FDC and post-FDC eras, 2-tailed alpha }=0.05 \text {. } \\
\text { *** NS" (not significant) defined as } P>0.05 \text {. }\end{array}$} \\
\hline \multicolumn{5}{|l|}{ 'Includes index dates $\geq 7 / 1 / 1999$ and $\leq 12 / 31 / 2004$. } \\
\hline \multicolumn{5}{|l|}{$\begin{array}{l}{ }^{2} \text { Includes index dates } \geq 1 / 1 / 2005 \text { and } \leq 6 / 30 / 2006 \\
{ }^{3} \text { Column percentages may not add up to } 100 \% \text { due to rounding. }\end{array}$} \\
\hline \multicolumn{5}{|l|}{${ }^{3}$ Column percentages may not add up to $100 \%$ due to rounding. } \\
\hline \multicolumn{5}{|c|}{${ }^{4}$ Baseline lab values (CD4, HIV RNA) include valid test closest to index date. } \\
\hline \multicolumn{5}{|c|}{${ }^{5}$ Baseline disease assessments include ANY presence of disease (yes/no) between start date and index date. } \\
\hline \multicolumn{5}{|c|}{${ }^{6}$ Baseline provider variables calculated as of calendar year of index date. } \\
\hline \multicolumn{5}{|c|}{$\begin{array}{l}{ }^{7} \text { Total pill count per day calculated from start date through exit date. Formula }=\text { Sum (pills per day [both ARV and non ARV] } \times \text { day supply)/exit date }- \text { start date. } \\
{ }^{8} \text { Region-based aggregate ARV pill count calculated as of six-month time period of index date. Includes count of total pills per day/per patient upon first dispensing that meets } \\
\text { cART regimen among all HIV patients within each region. }\end{array}$} \\
\hline Abbreviations: FDC, fixed dose combinationl IQR, interq & & & & \\
\hline
\end{tabular}

HBV and/or HCV. There were some differences in clinical characteristics between the two FDC-time eras. The distribution of CD4 count/HIV RNA level and of proportion meeting AIDS criteria differed significantly between the two FDC time eras. No other clinical differences were noted at baseline.

\section{Provider characteristics}

As of index date, most patients were assigned to providers with the following characteristics: 12 or more years of HIV experience (55.3\%) and HIV panel sizes greater than 100 (57.0\%) (Table 2). The distributions of years of HIV experience were significantly different between the two FDC-time eras; however, no differences were reported for HIV panel size.

\section{Treatment regimen characteristics}

The patient-based median total pill count was 1.7 pills per day overall, dropping from 1.8 pills per day in the pre-FDC era to 1.3 pills per day in the post-FDC era (Table 2). The region-based median ARV pill count (upon first cART dispensing for all HIV patients) was 13.0 pills per day overall, dropping from 18.0 pills per day in the pre-FDC era to 6.0 pills per day in the post-FDC era. 


\section{Results of the Cox regression analysis}

A total of 2,069 of the 2,127 patients (97.3\%), representing 1,272 events, had complete information available for multivariate analysis (Table 3).

\section{FDC treatment era}

The introduction of once-daily, fixed-dose combination therapies (FDC era) was not significantly associated (hazard ratio $[\mathrm{HR}]=0.92,95 \%$ confidence interval $[\mathrm{CI}]$ : 0.79-1.08) with initiation of cART, after adjusting for clinical, provider, treatment regimen, and demographic characteristics.

\section{Clinical characteristics}

Clinical characteristics were the strongest predictors of cART initiation. Compared to CD $4 \leq 200$ cells $/ \mu \mathrm{L}$ (reference category), the following characteristics were associated with a decreased likelihood of cART initiation: 1) CD4 201-350 cells $/ \mu \mathrm{L}$ and HIV RNA $<100,000(\mathrm{HR}=0.76$, 95\% CI: 0.62-0.94), 2) CD4 > 350 cells/ $\mu \mathrm{L}$ and HIV RNA

Table 3 Association of FDC treatment era status, clinical, provider, and treatment regimen characteristics with initiation of cART $(\mathrm{N}=2,069)^{\prime}$

\begin{tabular}{|c|c|c|}
\hline Characteristic & Hazard ratio & $95 \% \mathrm{Cl}$ \\
\hline \multicolumn{3}{|l|}{ FDC time era } \\
\hline Pre period (Ref. group) & 1.00 & NA \\
\hline Post period & 0.92 & $0.79-1.08$ \\
\hline \multicolumn{3}{|l|}{ Clinical } \\
\hline \multicolumn{3}{|l|}{ 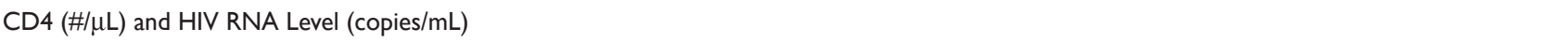 } \\
\hline$C D 4 \leq 200$ & 1.00 & NA \\
\hline CD4 20I-350 and HIV RNA $\geq 100 \mathrm{~K}$ & 1.24 & $0.95-1.62$ \\
\hline CD4 20I-350 and HIV RNA $<100 \mathrm{~K}$ & 0.76 & $0.62-0.94$ \\
\hline $\mathrm{CD} 4>350$ and $\mathrm{HIV}$ RNA $\geq 100 \mathrm{~K}$ & 0.69 & $0.5 I-0.93$ \\
\hline CD4 $>350$ and HIV RNA $<100 \mathrm{~K}$ & 0.15 & $0.12-0.20$ \\
\hline No AIDS-defining illness (Ref. group) & 1.00 & NA \\
\hline AIDS-defining illness & 2.05 & $1.68-2.50$ \\
\hline No active HCV and/or HBV (Ref. group) & 1.00 & NA \\
\hline Active HCV and/or HBV & 0.97 & $0.78-1.19$ \\
\hline \multicolumn{3}{|l|}{ Provider } \\
\hline \multicolumn{3}{|l|}{ Years caring for HIV patients } \\
\hline 0-5 (Ref. group) & 1.00 & NA \\
\hline $6-11$ & 1.08 & $0.92-1.26$ \\
\hline $12-16$ & 1.09 & $0.95-1.25$ \\
\hline$>16$ & 1.31 & $1.02-1.68$ \\
\hline \multicolumn{3}{|l|}{ HIV panel size } \\
\hline$\leq 100$ (Ref. group) & 1.00 & NA \\
\hline $101+$ & 1.01 & $0.90-1.13$ \\
\hline \multicolumn{3}{|l|}{ Treatment regimen } \\
\hline \multicolumn{3}{|l|}{ Total pill count per day (Patient-based) } \\
\hline$\leq$ Median (Ref. group) & 1.00 & NA \\
\hline$>$ Median & 1.56 & $1.38-1.77$ \\
\hline \multicolumn{3}{|l|}{ ARV pill count per day (Region-based) } \\
\hline$\leq$ Combined region median (Ref. group) & 1.00 & NA \\
\hline$>$ Combined region median & 1.15 & $0.94-1.40$ \\
\hline
\end{tabular}

'The effective sample size for the Cox regression (because of missing data) was 2,069 . Models adjusted for age ( $\leq$ median $=$ ref. group vs $>$ median), gender (female $=$ ref. group vs male), race/ethnicity (white = ref. group vs non-white ethnicity/other/unknown), IDU risk category (no = ref. group vs yes/unknown), and region (Non-California regions $=$ ref. group vs California region).

Abbreviations: ARV, antiretroviral; $\mathrm{HCV}$, hepatitis $\mathrm{C} ; \mathrm{HBV}$, hepatitis $\mathrm{B}$. 
$\geq 100,000$ copies $/ \mathrm{mL}$ ( $\mathrm{HR}=0.69,95 \% \mathrm{CI}: 0.51-0.93$ ), and

3) $\mathrm{CD} 4>350$ cells $/ \mu \mathrm{L}$ and HIV RNA $<100,000$ copies $/ \mathrm{mL}$ $(\mathrm{HR}=0.15,95 \%$ CI: 0.12-0.20). Patients who met AIDS criteria were more likely to receive cART (HR $=2.05,95 \%$ CI: 1.68-2.50), compared to patients who did not meet AIDS criteria.

\section{Provider characteristics}

Compared to patients assigned to providers with $0-5$ years of experience caring for HIV patients in the KP system, patients assigned to providers with $>16$ years of experience were more likely to initiate cART ( $\mathrm{HR}=1.31,95 \%$ CI: 1.02-1.68). HIV panel size was not significantly associated with likelihood of cART initiation.

\section{Treatment regimen characteristics}

Patients with total pill count per day above the group median were more likely to initiate cART ( $\mathrm{HR}=1.56,95 \%$ CI: 1.38-1.77), compared to patients with total pill count per day below the group median. Aggregate, region-based ARV pill count upon first cART dispensing was not significantly associated with likelihood of cART initiation.

\section{Discussion}

We found that implementation of once-daily FDC treatment regimens into clinical practice did not significantly affect initiation of cART. The finding that implementation of once-daily FDC treatment regimens was not independently associated with cART initiation is significant for HIV care. These results are significant because of potential concerns that the implementation of new drug combinations, while offering many patient advantages, may unduly influence provider prescribing behavior, independent of important clinical characteristics. Our findings showed this not the case. Rather, we found that KP clinicians' prescribing practices were independent of new drug combinations. Consistent with treatment guidelines, clinical factors such as CD4 T-cell count, HIV/RNA levels, and AIDS criteria were the strongest predictors of cART initiation. This is the first study we know of to examine the independent effect of implementation of once-daily FDC treatment regimens on initiation of cART.

Of particular interest from a health-care delivery perspective, we found that providers with 16+ years of experience caring for HIV patients were more likely to initiate cART, compared to providers with $0-5$ years of experience. These results confirm other research findings ${ }^{9}$ demonstrating benefits to HIV patients who have more experienced providers. This study finding has important implications for policy and practice. Our results suggest that within KP, ARV-naïve HIV patients assigned to providers with longer experience treating HIV patients may benefit from more appropriate pharmacotherapy such as earlier cART initiation.

We also found that higher total pill count per day was associated with initiation of cART. This may be because higher pill counts are potentially related to more advanced disease stage, disease burden, and/or co-morbidities, which may cause clinicians to be more aggressive in treatment, as there is some evidence that greater HIV viral control leads to improved health generally. Indeed, in the overall study population, $56 \%$ of patients either had a CD4 T-cell count $\leq 200$ cells $/ \mu \mathrm{L}(37 \%)$, or CD4 T-cell count $\geq 201$ to 350 cells $/ \mu \mathrm{L}$ with $\mathrm{HIV}-1$ RNA $\geq 100,000$ copies $/ \mathrm{mL}$ (Table 2), indicating a substantial proportion of individuals with more advanced disease and/or a more compelling need to initiate therapy, as per treatment guidelines. ${ }^{2,3,15}$ In contrast, however, higher overall pill count was associated with cART initiation, even after adjusting for CD4 T-cell count and HIV-1 RNA levels, the primary surrogate markers for assessing disease stage. Thus, more research is needed to fully interpret the association of total overall pill count per day with initiation of cART.

This study had several limitations. First, we could not capture physician orders to place patients on cART rather than pharmacy dispensings for cART. Measuring physician orders for cART would likely be a more sensitive method of detecting changes in initiation of cART after implementation of the once-daily FDC treatment era. Second, we were not able to measure years of provider experience caring for HIV patients prior to employment with KP. In addition, the finding that years of provider experience was associated with cART initiation cannot be generalized beyond KP. Third, limited follow-up time in the once-daily post-FDC era (about 1.5 years vs 5.5 years in the pre-FDC era) reduced our ability to detect differences in cART initiation in the post-FDC era. Fourth, we had few measures of patient-level socioeconomic status, such as educational level and income, available for analysis. Last, our study results may not be generalizable to nongroup-model HMO settings.

Our study showed that CD4 T-cell count, HIV RNA levels and meeting AIDS criteria were the strongest predictors of cART initiation. This result is very encouraging because it indicates that new, more convenient drug combinations do not necessarily unduly influence provider prescribing behaviors, independent of patient and clinical characteristics. Future research is needed to understand the effect of initiation of the once-daily FDC treatment era on patient outcomes among 
an HIV-infected ARV-naïve population. Specifically, more research is needed to understand whether the advent of the oncedaily FDC treatment era affected patient treatment-adherence levels, quality-of-life outcomes, and morbidity and mortality.

\section{Disclosure}

We would like to thank Gilead Sciences Corporation for providing investigator-sponsored funding to complete this research. None of the authors have institutional or commercial affiliations that would post a conflict of interest.

\section{References}

1. Panel on Clinical Practice for Treatment of HIV Infection. Guidelines for the use of antiretroviral agents in HIV-infected adults and adolescents. Afr J Med Pract. 1998;5:79-104.

2. Guidelines for the use of antiretroviral agents in HIV-infected adults and adolescents. Department of Health and Human Services and Henry J. Kaiser Family Foundation. MMWR Recomm Rep. 1998;47(RR-5):43-82.

3. Dybul M, Fauci AS, Bartlett JG, Kaplan JE, Pau AK; Panel on Clinical Practices for Treatment of HIV. Guidelines for using antiretroviral agents among HIV-infected adults and adolescents. Ann Intern Med. 2002;137(5 Pt 2):381-433.

4. Dybul M, Fauci AS, Bartlett JG, Kaplan JE, Pau AK; Panel on Clinical Practices for Treatment of HIV. Guidelines for using antiretroviral agents among HIV-infected adults and adolescents. Recommendations of the Panel on Clinical Practices for Treatment of HIV. MMMR Recomm Rep. 2002;51(RR-7):1-55.

5. Tashima KT, Mitty JA. Once-daily therapies for the treatment of HIV infection. HIV/AIDS Rep. 2006;3:86-92.

6. Carey D. Once-daily therapies. J HIV Ther. 2003;8:7-11.
7. Stone VE, Jordan J, Tolson J, et al. Perspectives on adherence and simplicity for HIV-infected patients on antiretroviral therapy: self-report of the relative importance of multiple attributes of highly active antiretroviral therapy (HAART) regiments in predicting adherence. J Acquir Immune Defic Syndr. 2004;36:808-816.

8. Sherer RD, Fath MJ, Da Silva BA, et al. The importance of potency and durability in HIV patient antiretroviral therapy preferences: A telephone survey. AIDS Patient Care STDS. 2005;19:794-802.

9. Kitahata M, Van Rompaey S, Shield A. Physicians' experience in the care of HIV-infected persons is associated with earlier adoption of new antiretroviral therapy. J Acquir Immune Defic Syndr. 2000;24:106-114.

10. Piacenti FJ. An update and review of antiretroviral therapy. Pharmacotherapy. 2006;26:1111-1133.

11. Sax PE, Gathe Jr JC. Beyond efficacy: the impact of combination antiretroviral therapy on quality of life. AIDS Patient Care STDS. 2005; 19:563-576.

12. Patterson DL, Windells S, Mohr J, et al. Adherence to protease inhibitor therapy and outcomes in patients with HIV infection. Ann Intern Med. 2000;133:21-30.

13. Phillips AN, Miller V, Sabin C, et al. Durability of HIV-1 viral suppression over 3.3 years with multi-drug antiretroviral therapy in previously drug-naïve individuals. AIDS. 2001;15:2379-2384.

14. Tashima, KT, Hogan JF, Gardner LI, et al. A longitudinal analysis of hospitalization and emergency department use among HIV-infected women reporting protease inhibitor use. Clin Infec Dis. 2001;33: 2055-2060.

15. Hammer SM, Saag MS, Schecter M, et al. Treatment of adult HIV Infection: 2006 recommendations of the International AIDS Society-USA panel. JAMA. 2006;296:827-843.

16. Kitihata MM, Gange SJ, Moore RD. Initiation Rather than Deferring HAART at a CD4 Count Between 351-500 Cells $/ \mathrm{mm}$ is Associated with Improved Survival. Washington, D.C.: Presented at 46th Annual Interscience Conference on Antimicrobial Agents and Chemotherapy; 2008 .
HIV/AIDS - Research and Palliative Care

\section{Publish your work in this journal}

HIV/AIDS - Research and Palliative Care is an international, peerreviewed open-access journal focusing on advances in research in HIV, its clinical progression and management options including antiviral treatment, palliative care and public healthcare policies to control viral spread. The journal welcomes original research, basic science,

\section{Dovepress}

clinical \& epidemiological studies, reviews \& evaluations, expert opinion \& commentary, case reports \& extended reports. The manuscript management system is completely online and includes a very quick and fair peer-review system. Visit http://www.dovepress.com/ testimonials.php to read real quotes from published authors. 